\title{
Beiträge zur Muskelchemie der Octopoden.
}

\author{
Von
}

\section{Henze.}

(Aus dem chemischen Laboratorium der zoologischen Station zu Neapel.)

(Der Redaktion zugegangen am 9. Januar 1905.)

Unsere Kenntnisse in bezug auf die chemischen Verhältnisse der Muskulatur wirbelloser Tiere überschreiten kaum die ersten Anfänge. Die Literatur ${ }^{1}$ ) verfügt auf diesem Gebiet über eine größere Anzahl zerstreuter oder zufällig gemachter Beobachtungen, die an sich vielfach noch einer exakten Prüfung warten. Schon jetzt läßt sich jedoch feststellen; daß wir durchaus nicht berechtigt sind, die Vorstellungen, die wir uns über die Zusammensetzung und über den Stoffwechsel des quergestreiften Wirbeltiermuskels gebildet haben, ohne weiteres auf die Wirbellosen zu übertragen. Ebenso unbegründet ist die Verallgerneinerung der bei einzelnen Wirbellosen gefundenen Daten auf ganze Klassen. Der Weg führt auch hier vom Speziellen zum Allgemeinen. Eine Erweiterung unserer Kenntnisse in obengenannter Richtung und in den damit zusammenhängenden Fragen muß zunächst, einmal durch systematische Untersuchungen der einzelnen Tierspezies angebahnt werden. Die vorliegende Mitteilung beschäftigt sich etwas eingehender mit der Muskelchemie der Octopoden.

Histologisch scheint die Muskulatur der Octopoden eine Mittelstellung zwischen glatter und quergestreifter Muskulatur einzunehmen. Mit dem Studium der Eiweißkörper des Octopus-

1) Vergl. besonders die Arbeiten von Kruckenberg, die neben der übrigen Literatur bei v. Fürth: «Vergleichende chemische Physiologie der niederen Tiere zitiert sind.

Hoppe-Seyler's Zeitschrift f. physiol. Chemie. XLIII. 
muskels hat sich v. Fürth ${ }^{1}$ ) in gewisser Richtung beschäftigt. Dagegen fehlt bisher eine eingehendere Untersuchung der sogenannten Extraktiv- und Reservestoffe. Wir müssen über diese Substanzen unterrichtet sein, nicht nur um einen Einblick in die chemischen Prozesse, welche sich im Muskel abspielen, zu gewinnen, sondern auch um überhaupt ein deutlicheres Bild vom gesamten Stoffwechsel dieser Tiere zu erhalten. Naturgemäß stehen diese Verbindungen in nächster Beziehung zu den Exkretionsprodukten und es war um so eher zu hoffen, nach dieser Seite Zusammenhänge zu finden, da über die Chemie der Harnsekretion der Octopoden bereits ebenfalls von v. Fürth ${ }^{2}$ ) eine Arbeit vorliegt. v. Fürth zeigte, daß ein sehr großer Teil des Stickstoffs im Harn in Form von Ammoniak eliminiert wird. Daneben findet sich viel Hypoxanthin und nicht unbeträchtliche Mengen einer stickstoffhaltigen Verbindung von sauerem Charakter, deren Konstitution bisher noch nicht aufgeklärt werden konnte. Harnsäure tritt höchstens in Spuren auf, während Harnstoff, Kreatin und Hippursäure vollständig fehlen. Nach den hier nur angedeuteten unverkennbaren Abweichungen von unseren sonstigen Erfahrungen dürfen wir auch auf einen in wesentlich anderer Weise verlaufenden Stoffwechsel schließen. Es war meine Absicht, neben einigen quantitativen Untersuchungen in vorliegender Studie speziell den Extraktiv- und Reservestoffen des Muskels meine Aufmerksamkeit zu schenken, wobei von einer erschöpfenden Behandlung der Aufgabe natürlich nicht die Rede sein konnte.

Zur Untersuchung wurde die Muskulatur der Arme und des Mantels benutzt und zwar sofort nach der Verblutung der Tiere. Die Haut wurde entfernt und die Muskeln mit einer guten Fleischhackmaschine fein zerkleinert. Die Tiere selbst wurden vor der Tötung längere Zeit im Aquarium gehalten und gut gefüttert, sodaß sie sich stets in bestem Ernährungszustand befanden.

1) v. Fürth, Diese Zeitschrift, Bd. XXXI, S. 338 .

2) v. Fürth, Diese Zeitschrift, Bd. XXXI, S. 353. 


\section{Die stickstoffhaltigen Bestandteile.}

Der Gesamtstickstoffgehalt des Muskels ist ein wenig niedriger, als er im allgemeinen für die Wirbeltiere angegeben wird. Er beträgt im Mittel

$13,13 \%$ berechnet auf Trockensubstanz,

$$
3,03 \% \text { > frisches Fleisch. }
$$

1. $0,3188 \mathrm{~g}$ Trockensubstanz gaben nach Kjeldahl $0,0420 \mathrm{~g} \mathrm{~N}$

\section{0,3432 > > > 0,0449 , ,}

Die große Zahl der Muskelextrakte, die im Laufe der Untersuchung nötig waren, wurden in der Weise bereitet, daß der Muskelbrei (es kamen jedesmal 1,5-2 kg zur Verwendung). mit warmem Wasser übergossen, unter Umrühren einige Zeit stehen gelassen und hierauf scharf abgepreßt wurde. Diese Operation wurde mindestens dreimal mit neuen Wassermengen wiederholt. Die so erhaltenen Flüssigkeiten wurden unter Zusatz von etwas festem Aluminiumsulfat oder Alaun kurz aufgekocht und die Eiweißkörper koaguliert, was bisher nur auf diese Weise glatt gelang. Das Filtrat wurde zunächst in Gegenwart von etwas Magnesiumkarbonat, also bei neutraler Reaktion, eingeengt, und hierauf in den meisten Fällen mit Baryumhydroxyd ausgefällt. Der Überschuß des letzteren wurde durch Einleiten von Kohlensäure entfernt und die eventuell mit Essigsäure neutralisierte Lösung stark konzentriert und zur Kristallisation gestellt.

Schon nach kurzer Zeit findet man einen solchen Sirup vollkommen durchsetzt von einem Kristallbrei, der abgesaugt und mit verdünntem Alkohol nachgewaschen, sich als fast reines Taurin erweist. Die Substanz zeigt die charakteristischen Kristallformen und Reaktionen des Taurins. Bei der Analyse lieferten:

$$
\begin{gathered}
0,2454 \mathrm{~g} \text { Substanz 0,4534 } \mathrm{g} \mathrm{BaSO}_{4}=0,0624 \mathrm{~g} \mathrm{~S} \text {. } \\
\text { Gefunden: } 25,44 \% \mathrm{~S} \\
\text { Berechnet: } 25,60 \% \text {. }
\end{gathered}
$$

In einigen Fällen wurde das isolierte Taurin gewogen. Es gaben:

$$
\begin{aligned}
& 2 \mathrm{~kg} \text { frischer Muskel } 10,0 \mathrm{~g} \text { Taurin } \\
& 1,10>\quad \text { > } \\
& 2,20>\quad \text { > }
\end{aligned}
$$


Im Mittel enthält also der Muskel die große Menge von mindestens $0,5 \%$ Taurin.

Das Vorkommen des Taurins in den Muskeln der Octopoden ist bereits durch Valenciennes und Frémy ${ }^{1}$ ) festgestellt worden. Auch wurde das Taurin durch die Analyse als solches identifiziert. Aus dem folgenden ergibt sich, daß das Taurin weitaus die größte Menge der stickstoffhaltigen Extraktivstoffe ausmacht. Um so auffallender ist es, daß v. Fürth keine Spur dieser Verbindung im Harn der Tiere nachzuweisen vermochte, was auch ich bestätigen kann. Das Taurin scheint also ein spezifisches Stoffwechselprodukt des Muskels zu sein, das vor seiner Ausscheidung vielleicht weiterer Oxydation unterliegt. In der Leber der Tiere konnte ich Taurin gleichfalls nachweisen.

Im Wirbeltiermuskel spielt das Kreatin die wichtigste Rolle unter den stickstoffhaltigen Extraktivstoffen. Wir vermissen diese Verbindung im Octopusmuskel vollständig. Es wurde danach in den Mutterlaugen der Taurinkristallisation gesucht. $\mathrm{Da}$ auch bei längerem Stehen derselben in der Kälte keine auf Kreatin deutende Kristallabscheidung eintrat, wurden mehrmals größere Teile der Mutterlaugen mit Salzsäure gekocht um etwa vorhandenes Kreatin in Kreatinin überzuführen. In den so vorbereiteten Flüssigkeiten versagte jedoch in jedem Falle sowohl die Reaktion von Weyl mit Nitroprussidnatrium als auch die Jaffésche Reaktion mit Pikrinsäure.

In einem anderen, zu diesem Zweck hergestellten Muskelextrakt wurde die Abscheidung der genannten Verbindung nach den Angaben von Maschke ${ }^{2}$ ) probiert. Nach Auskristallisation des Taurins wurden die Mutterlaugen mit Kupferoxydul behandelt und die ausgefällten Purinkörper zunächst entfernt. Im Filtrat hätte sich das Kreatinin in Form seiner Kupferoxydulverbindung in Lösung befinden müssen, die durch Sättigen mit Soda zur Ausfällung kommen mußte. Nähere Prüfung zeigte, daß dies nicht der Fall war.

1) Valenciennes u. Frémy, C. R., Bd. 41, S. 739. Vergl. auch L. Frédéricq, Arch. de Zool. expérim., Bd. VII, S. 582.

s) Maschke, Z. f. analyt. Chem., Bd. 17, S. 134. 
In Übereinstimmung mit diesen negativen Befunden steht auch die Beobachtung von v. Fürth, dem es in keiner Weise gelang, das Kreatin im Harn der Cephalopoden aufzufinden. Das Kreatin nimmt demnach augenscheinlich überhaupt nicht am Stoffwechsel der Octopoden teil.

Zur Prüfung der Muskelextrakte auf Purinbasen wurden die mit Wasser verdünnten Taurinmutterlaugen bei Gegenwart von überschüssigem Ammoniak mit ammoniakalischer Silberlösung ausgefällt. Der abzentrifugierte und mehrfach mit verdünntem Ammoniak gewaschene Niederschlag wurde aus Salpetersäure (spez. Gew. 1,1) unter Zusatz von Harnstoff umkristallisiert. Die sogenannte «Hypoxanthin»fraktion schied sich dabei in schönen Nadeln ab und wurde ein zweites Mal aus Salpetersäure umkristallisiert. Die Mutterlaugen gaben bei Übersättigen mit Ammoniak nur eine Trübung, sodaß das Vorkommen nur einigermaßen nennenswerter Mengen von Xanthin ausgeschlossen scheint. Die Hypoxanthinsilberfraktion wurde mit Schwefelwasserstoff zerlegt. Beim Einengen fiel das Hypoxanthin aus. Ein Teil davon wurde mit negativem Erfolg mittels Metaphosphorsäure auf Guanin geprüft. Zur Analyse wurde das Hypoxanthin mit überschüssigem Silbernitrat und Ammoniak in der Hitze in die Silberoxydverbindung verwandelt. Von der gut gewaschenen und bei $110^{\circ}$ getrockneten Verbindung lieferten

$$
\begin{aligned}
& 0,2210 \mathrm{~g}=0,1325 \mathrm{~g} \mathrm{Ag} .=59,96 \% \mathrm{Ag} \\
& \text { Gefunden }=50,18 \% \\
& \text { Berechnet für } \mathrm{C}_{5} \mathrm{H}_{2} \mathrm{Ag}_{2} \mathrm{~N}_{4} \mathrm{O}=60,0
\end{aligned}
$$

Aus $2 \mathrm{~kg}$ Muskel wurden 1,5 g Silberdoppelsalz erhalten. Da das letztere keine konstante Zusammensetzung hat, so läßt sich der Hypoxanthingehalt nur schätzungsweise auf ca. $0,03 \%$ angeben.

Es sei auf die schon anfangs erwähnte Tatsache hingewiesen, daß Hypoxanthin auch im Harn in reichlicher Menge ausgeschieden wird.

Anschließend an die Aufklärung der Natur der freien, aus dem Muskel extrahierbaren Purinkörper schien es wünschenswert eine Vorstellung über den Gesamtpurinbasengehalt des Muskels zu gewinnen. Die quantitative Bestimmung wurde nach 
der bekannten Methode von Burian und seinen Mitarbeitern ausgeführt. Über die Einzelheiten des Verfahrens, die streng innegehalten wurden, sei auf die letzte Arbeit von Burian und Walker Hall ${ }^{1}$ ) verwiesen. Es kamen 500 g Muskelbrei zur Verwendung, die durch 14 stündiges Kochen mit $4500 \mathrm{ccm}$ Wasser und $45 \mathrm{ccm}$ Schwefelsäure zerlegt wurden. Die Vorbereitung und Ausführung der sogenannten «Hauptfällung erfolgte genau nach genannter Vorschrift. Die sogenannte «Korrekturfällung» wurde ebenfalls ausgeführt, doch ließ sich aus der nach der Zerlegung des Bleiniederschlags resultierenden Flüssigkeit mit ammoniakalischer Silberlösung kein weiterer Purinbasenniederschlag mehr gewinnen.

Gesamtpurinbasen-Ag-Niederschlag (AgCl-haltig)

$$
\text { im Vacuum getrocknet }=2,0513 \mathrm{~g}
$$

D. h. umgerechnet auf AgCl-freie Substanz $=1,8577$,

$0,5660 \mathrm{~g} \mathrm{AgCl}$-haltige Substanz enthalten $0,0543 \mathrm{~g} \mathrm{AgCl}$, entsprechend 0,5127 g AgCl-freier Substanz.

1. $0,5309 \mathrm{~g}$ Substanz $=0,4906 \mathrm{~g} \mathrm{AgCl}$-freie Substanz liefern nach Kjeld a hl $0,0596 \mathrm{~g} \mathrm{~N}$

2. 0,4342 g Substanz $=0,3933$ g AgCl-freie Substanz liefern nach Kjeld a hl $0,0477 \mathrm{~g} \mathrm{~N}$.

Im Mittel berechnet sich daraus der Purinbasen-N aus $500 \mathrm{~g}$ Muskel auf $0,2277 \mathrm{~g}$.

Der Octopusmuskel enthält also 0,0456\% Purinbasen-N. Burian fand in verschiedenen Wirbeltiermuskeln 0,055-0,071\% Purinbasenstickstoff.

Durch Berechnung des Verhältnisses zwischen Ag- und N-Gehalt des Purinbasensilberniederschlags hat Burian auf die Natur der Purinkörper einen Rückschluß gemacht. Für die Niederschläge aus Pankreas ergaben sich Quotienten, die fast genau auf Guanin stimmten. Aus den obigen Daten berechnet sich das $\mathrm{N}-\mathrm{Ag}$-Verhältnis zu

$$
\frac{\mathrm{N}}{\mathrm{Ag}}=\frac{12,15}{49,36}
$$

Auf eine Einheitlichkeit der Muskelpurinkörper läßt sich hieraus nicht schließen. Eine eingehende Prüfung der Verhältnisse wurde nicht weiter verfolgt.

1) Burian u. Walker Hall, Diese Zeitschrift, Bd. XXXVIII, S. 336. 
Schon anfangs wurde erwähnt, daß der Harn der Octopoden harnstofffrei ist. Die Prüfung eines alkoholischen Muskelextraktes nach dieser Richtung gab gleichfalls ein negatives Resultat.

Die Möglichkeit, daß auch andere stickstoffhaltige Verbindungen, namentlich soweit dieselben als Eiweißzerfallsprodukte in Betracht kommen, unter den Extraktivstoffen des Muskels auftreten könnten, war nicht ohne weiteres ausgeschlossen. Es wurden daher zunächst einige von Taurin und Purinbasen befreite Muskelextrakte mit Phosphorwolframsäure bei saurer Reaktion gefällt, nachdem zuvor auch die Ammonsalze durch längeres Erwärmen mit Magnesiumoxyd vertrieben worden waren. Der Phosphorwolframsäureniederschlag war ziemlich stark. Er wurde nach der bekannten Kosselschen Vorschrift auf die Hexonbasen verarbeitet, ohne daß eine derselben nachgewiesen werden konnte. Zweifellos enthält der Phosphorwolframsäureniederschlag noch andere stickstoffhaltige Verbindungen. Es gelang, aus dem Phosphorwolframsäureniederschlag eine Base in Form ihres Platinsalzes zu isolieren. Dasselbe zersetzte sich jedoch beim Umkristallisieren. Meine vielfachen Bemühungen, die Verbindung näher zu charakterisieren, sind bisher vergeblich geblieben, sodaß ich von einer genauern Beschreibung der bisherigen Beobachtungen einstweilen absehe.

Im obigen Sinne habe ich auch im Muskel nach der von v. Fürth im Harn gefundenen stickstoffhaltigen Verbindung saueren Charakters gesucht. Dieselbe ist dadurch ausgezeichnet, daß sie fast nur durch Quecksilberacetat fällbar ist. Es gelang leicht, dieselbe aus dem Harn von Octopus zu gewinnen und einige ihrer Reaktionen zu studieren, sodaß damit zugleich die Angaben v. Fürths eine Bestätigung finden. Die purinfreien Muskelextrakte geben nach vorheriger Ausfällung mit Bleiacetat und Entfernung des Bleies allerdings Niederschläge mit Quecksilberacetat, doch hat-deren Weiterverarbeitung vorderhand noch zu keinem positiven Resultat geführt.

Weitere Prüfungen galten dem eventuellen Vorkommen gewisser Amidosäuren. Für den Wirbeltiermuskel kann es als sicher gelten, daß derselbe, solange er frisch und keinen auto- 
lytischen oder pathologischen Prozessen verfallen ist, keine Amidosäuren enthält. Dagegen ist z. B. für die Muskulatur einiger Mollusken schon früher und auch neuerdings wieder festgestellt worden, daß dieselben nicht unbeträchtliche Mengen von Glykokoll beherbergen. Chittenden fand zuerst bei Pecten irradians 0,39 bis $0,71 \%$ Glykokoll. ${ }^{1}$ ) Die Angaben einiger Autoren über das Vorkommen von Tyrosin z. B. bei Eledone, dem nächsten Verwandten von Octopus, sowie bei Krebsen, darf man wohl vorderhand noch mit einem Fragezeichen versehen. Vergl. darüber die Bemerkungen bei v. Fürth. ${ }^{2}$ )

Für die genannten Zwecke wurden die Extrakte, von denen einige zuvor mit Phosphorwolframsäure gefällt worden waren, nach Entfernung der überschüssigen Phosphorwolframsäure zum Teil mit frischgefälltem Kupferhydroxyd gekocht, um die Abscheidung schwerlöslicher Kupfersalze zu versuchen. Andere Portionen wurden in alkalischer Lösung mit $\alpha$-Naphtolsulfochlorid geschüttelt, um zu prüfen, ob hierbei eine Abscheidung der entsprechenden schwerlöslichen Kondensationsprodukte zu erreichen wäre. Die Versuche gaben stets negative Resultate.

Nach den schon mehrfach erwähnten Untersuchungen von v. Fürth wird ein auffallend großer Teil des Stickstoffs im Harn des Cephalopoden in Form von Ammoniakstickstoff eliminiert. Im Vergleich mit den Verhältnissen beim Hunde ist der Betrag ca. viermal so groß.

Im Hinblick hierauf war es nicht ausgeschlossen, daß der Muskel in seinem Stoffwechsel größere Quantitäten von Ammonsalzen bildet, umsomehr als das Kreatin fehlt. Neuere Untersuchungen über den Ammoniakgehalt tierischer Organe und der Muskeln verdanken wir Nencki und seinen Mitarbeitern. Es wurden mit Hilfe der von ihnen ausgearbeiteten Methode, die auf der Destillation der Organe mit Magnesiumoxyd im Vacuum beruht, ${ }^{3}$ ) zwei quantitative Bestimmungen des Ammoniakgehaltes des Octopusmuskels ausgeführt.

1) Chittenden, Ann. Chem. u. Pharm., Bd. 178, S. 266.

Vergl. auch A. Kelly, Hofmeisters Beiträge, Bd. V, S. 380.

2) G. v. Fürth, Vergleichende chem. Physiol. d. nied. Tiere, S. 438.

s) Nencki und Zaleski, Diese Zeitschrift, Bd. XXXIII, S. 193. 
I. $50 \mathrm{~g}$ frischer Muskel gaben ein Destillat, das $2,20 \mathrm{ccm} \mathrm{n} / 10 \mathrm{H}_{2} \mathrm{SO}_{4}$ sättigte, d. h. der Muskel enthielt in Milligrammen 7,48\% $\mathrm{NH}_{3}$.

II. $50 \mathrm{~g}$ Muskel des gleichen Tieres lieferten ein Destillat, welches $2,40 \mathrm{ccm} \mathrm{n} / 10 \mathrm{H}_{2} \mathrm{SO}_{4}$ sättigte, d. h. der Muskel enthielt in Milligrammen $8,16 \% \mathrm{NH}_{8}$.

Die Destillation wurde ca. 5 Stunden lang im Gang gehalten bei $37-39^{\circ}$.

Die gefundenen Werte sind im Vergleich mit den für den Hundemuskel vorliegenden eher als niedrig zu bezeichnen.

Auf ein besonderes Produktions- oder Retentionsvermögen von Ammonsalzen im Octopusmuskel dürfte deshalb ohne weiteres nicht zu schließen sein.

\section{Die stickstofffreien Bestandteile.}

Der quergestreifte Muskel enthält stets mehr oder minder große Mengen von Fleischmilchsäure, die sowohl bei erhöhter Tätigkeit als beim Absterben des Muskels zunehmen. Der tote Muskel reagiert deshalb sauer. Man überzeugt sich leicht, daß auch der Octopusmuskel im Leben alkalisch oder amphoter reagiert, um beim Absterben schwach saure Reaktion anzunehmen. Für Wirbellose habe ich vergebens in der Literatur gesucht, etwas auf Milchsäure Bezügliches zu finden. Es mußte also im vorliegenden Fall zunächst einmal die Frage geklärt werden, ob Fleischmilchsäure überhaupt ein Muskelstoffwechselprodukt der Octopoden ist.

Während im Wirbeltiermuskel die Milchsäure ohne Schwierigkeiten $\mathrm{zu}$ bestimmen ist, da ihre Menge bis zu $1 \%$ (zitiert nach Neumeisters Lehrbuch) beträgt, wollte es anfangs nicht gelingen, die Säure im Muskelfleisch der Octopoden nachzuweisen. Die Methode der Darstellung wurde mehrfach variiert, z. B. auch die Extraktion des Muskels mit Alkohol nach Heffter ${ }^{1}$ ) versucht. Trotz der anfänglichen Mißerfolge war es dennoch schließlich möglich, eine wenn auch nur sehr geringe Menge Milchsäure nachzuweisen. Wie aus dem folgenden hervorgeht, handelt es sich jedoch dabei nicht um wirkliche Fleischmilchsäure.

1) Heffter, Arch. f. experim. Pathol. u. Pharm., Bd. 38. 
Der wässerige Extrakt von $1125 \mathrm{~g}$ Muskelbrei wurde unter Alaunzusatz koaguliert und nach Filtration mit Barythydrat ausgefällt. Nachdem der Barytüberschuß durch Einleiten von Kohlensäure entfernt und die Flüssigkeit bis nahe zum Sirup eingeengt worden war, wurde der letztere in der Wärme mit ca. der 10 fachen Menge Alkohol gefällt. Der Alkohol wurde nach dem Erkalten abgegossen und der Rückstand mit Alkohol nachgewaschen. Der nach dem Abdestillieren des Alkohols verbleibende Rückstand wurde in etwas Wasser aufgenommen und mit zirka dem gleichen Volumen 50\% iger Phosphorsäure versetzt und mit großen, mehrfach erneuten Äthermengen ausgeschüttelt, Es bilden sich hierbei stets sehr lästige Emulsionen. Die vereinigten Ätherauszüge wurden abdestilliert. Der Rückstand gab die Uffelmannsche Reaktion und wurde in wässeriger Lösung mit $\mathrm{ZnCO}_{3}$ gekocht. Das eingeengte Filtrat lieferte neben geringen Mengen eines Sirups glänzende, in Rosetten angeordnete prismatische Kristalle, die nach Entfernung des verunreinigenden Sirups durch Alkohol aus Wasser umkristallisiert wurden. Die Menge der reinen Kristalle betrug nur 0,26 g aus über $1 \mathrm{~kg}$ Muskel.

$0,1921 \mathrm{~g}$ lufttrockener Substanz verloren bei $100^{\circ} 0,0344 \mathrm{~g} \mathrm{H}_{2} \mathrm{O}$,

d. i. $17,85 \%$ Kristallwasser.

Gärungsmilchsäure verlangt $18,18 \%$ Kristallwasser

Fleischmilchsäure > $12,9 \%$

$0,1577 \mathrm{~g}$ wasserfreies Salz lieferten $0,0525 \mathrm{~g} \mathrm{Zn0}$

Gofundon: $26,75 \% \mathrm{Zn}$

Berechnet: $26,75 \%$,

Die Analyse beweist also, daß auch im Octopusmuskel Milchsäure auftritt. Allerdings wurde nur die geringe Menge von $0,014 \%$ isoliert. Dieses Ergebnis wird dadurch noch auffallender, als, nach dem Kristallwassergehalt des Zinksalzes zu schließen, die Säure Gärungsmilchsäure ist. Übrigens sprach auch die Unlöslichkeit dieses Salzes in Alkohol dafür.

Nach Untersuchungen von Heintz soll auch im quergestreiften Muskel etwas Gärungsmilchsäure $\mathrm{zu}$ finden sein, und nach Gscheidlen ${ }^{1}$ ) ist die Milchsäure des Gehirns, wie auch schon Müller fand, Gärungsmilchsäure. Der Fund wäre

1) Gscheidlen, Pflügers Archiv, Bd. 8, S. 171. 
also nicht ganz ohne Analogie. Die im Darm und Magen auftretende inaktive Gärungsmilchsäure kann hier natürlich nicht zum Vergleich herangezogen werden. Einstweilen scheint der Schluß gerechtfertigt, daß die Milchsäure bei den Octopoden bei weitem nicht die Rolle im Muskelstoffwechsel spielt, wie dies bei den Wirbeltieren der Fall ist. Die eigentliche Fleischmilchsäure scheint vollständig zu fehlen und es dürfte nicht ausgeschlossen sein, daß die sehr geringen Mengen von Gärungsmilchsäure mit dem Muskelstoffwechsel direkt gar nichts zu tun haben.

Der wichtigste stickstofffreie Bestandteil des Wirbeltiermuskels ist das Glykogen. Neumeister sagt in seinem Lehrbuch, das Glykogen sei in den Muskeln der Tiere aller Klassen und Spezies nachweisbar. Es scheint mir durchaus zweifelhaft, $\mathrm{ob}$ diese Behauptung bei unserem lückenhaften Wissen in der vergleichenden Muskelchemie aufrecht zu halten ist. Speziell in bezug auf den vorliegenden Fall fand ich eine Bemerkung Frédéricqs ${ }^{1}$ ) in seiner bekannten Arbeit: "Recherches sur la physiologie du poulpe commun». Er sagt, daß ein von ihm geprüfter Muskelextrakt kein Glykogen $\mathrm{zu}$ enthalten schien. Jede nähere Angabe fehlt sonst.

Für die diesbezügliche Untersuchung wurde besonders Sorge getragen, daß sich die Tiere in bestmöglichem Ernährungszustande befanden und daß andererseits die Muskeln sofort nach dem Tode verarbeitet wurden. Für jeden Versuch kamen nie weniger als $1-1^{1 / 8} \mathrm{~kg}$ Muskel zur Verwendung. Unter Beachtung dieser Punkte habe ich die meisten Versuche zur Isolierung des Glykogens nach Brücke gemacht und ebenso zwei nach dem von Pflüger ${ }^{2}$ ) angegebenen Verfahren durch direktes Aufschließen des Muskelbreies mit 60\% iger Kalilauge. Hierbei wurden je $300 \mathrm{~g}$ Muskel benutzt. In keinem der zahlreichen Versuche ist es möglich gewesen, Glykogen aufzufinden. Selbst mit Hilfe der Jodreaktion ließ sich nicht der geringste Anhalt dafür gewinnen.

Auf Grund dieser stets negativen Resultate halte ich es für ausgeschlossen, daß die Cephalopoden einen Reservestoff

1) L. Frédéricq, Arch. de Zool. expérim., Bd. VII, S. 582.

2) Pflüger, Pflügers Archiv, Bd. 96, S. 1. 
in Form von Glykogen in den Muskeln beherbergen. Den möglichen Einwand, daß das Glykogen nur zu ganz bestimmten Perioden in den Geweben abgelagert wird, glaube ich ebenfalls zurückweisen zu können, da die Untersuchungen sowohl im Sommer wie im Winter angestellt wurden. Ferner sei nochmals hervorgehoben, daß die Tiere stets überreichlich gefüttert worden waren, und bei den großen Quantitäten Muskel, die jedesmal verarbeitet wurden, hätten sich doch wenigstens Spuren von Glykogen finden sollen. Daß die Tiere irgend einen Reservestoff haben, muß man schon daraus schließen, daß dieselben monatelang völlig ohne Nahrung leben können. Es dürfte das nächstliegende sein, an einen Ersatz des Glykogens durch irgend eine andere Substanz zu denken.

In den Muskelextrakten selbst konnte mit Sicherheit nicht eine direkt reduzierende Substanz nachgewiesen werden, ebensowenig wenn dieselben einige Zeit mit verdünnter Säure gekocht worden waren. Wäre es aber nicht möglich, daß z. B. Glykoproteide oder ähnliche Substanzen die Rolle des Glykogens übernehmen, falls es sich überhaupt um Verbindungen handelt, die den Kohlehydraten nahestehen?

Wenn man die bei Bereitung der Muskelextrakte restierenden Eiweißkörper mit 3\%iger Schwefelsäure 2-3 Stunden hydrolysiert und nach Filtration die Flüssigkeit mit Phosphorwolframsäure ausfällt und das mit Barythydrat behandelte Filtrat unter Einleiten von Kohlensäure einengt, so reduziert dasselbe sehr stark Fehlingsche Lösung. Ebenso gibt dasselbe beim Erhitzen mit Alkali starken Karamelgeruch und mit $\alpha$-Naphtol intensivste Reaktion. Nach dieser Beobachtung scheinen also im Muskel leicht Zucker abspaltende Proteinkörper vorzukommen. Ich bin mir wohl bewußt, daß mit dieser Angabe vorderhand noch wenig anzufangen ist.

Vorgreifend sei eine andere Beobachtung erwähnt. Meine Bemühungen, in dem Hepatopankreas der Octopoden Glykogen aufzufinden, sind bisher ebenfalls vergeblich gewesen. Nur soviel vermag ich zu behaupten, daß es gelang, aus wässerigen Leberauszügen nach Ausfällung mit Jodquecksilber-Jodkalium und Salzsäure durch Alkohol sehr geringe Mengen einer Substanz zu fällen, die zwar immer noch Biuretreaktion erkennen ließ, 
ohne jemals Braunfärbung mit Jodlösung zu zeigen. Nach kurzem Erwärmen der Substanz mit verdünnten Säuren erhält man jedoch eine Flüssigkeit, die stark Fehlingsche Lösung reduziert und nach noch nicht abgeschlossenen Versuchen auch mit Phenylhydrazin zu reagieren scheint.

Die Vermutung, ob nicht vielleicht das Fehlen des Glykogens und augenscheinlich auch der Fleischmilchsäure in einem inneren Zusammenhang stehen, dürfte nicht ganz unberechtigt sein.

\section{Wassergehalt und mineralische Bestandteile des Muskels.}

Der Wassergehalt des frischen Muskels wurde zu 77,31\% gefunden. 40,1 g frischer Muskel gaben beim Trocknen bei $100^{\circ}$ 9,10 g Trockensubstanz, d. i. in Prozenten 22,69 g.

Die im folgenden mitgeteilten quantitativen Bestimmungen der mineralischen Bestandteile des Muskels wurden hauptsächlich im Hinblick auf die Verteilung der Alkalien ausgeführt. Infolge der Lebensweise der Tiere in einem Milieu mit sehr hohem Gehalt an Natriumsalzen schien es fraglich, ob auch bei ihnen im Muskel die Kaliumsalze gegenüber den Natriumverbindungen überwiegen.

Wir verdanken $\mathrm{Katz}^{1}$ ) eine große Anzahl sehr sorgfältiger Untersuchungen über die mineralischen Bestandteile des Muskelfleisches verschiedener Wirbeltiere. Seine Erfahrungen dienten als Anhalt, sodaß fast ganz auf die von ihm verwandten und geprüften Methoden verwiesen werden kann. $\mathrm{Zu}$ den Analysen wurden $500 \mathrm{~g}$ Muskelbrei, die unter sorgfältigster Vermeidung von Verunreinigung mit Meerwasser hergestellt worden waren, nach Voits Methode ${ }^{2}$ ) unter Zusatz von Alkohol getrocknet, fein pulverisiert und für jede Einzelbestimmung die erforderliche Quantität bei $100^{\circ}$ zur Gewichtskonstanz gebracht. Die Verasehung erfolgte, je nach den eventuell zugesetzten Salzen, in Platin- oder Silbergefäßen.

In 100 Teilen trockener Muskelsubstanz wurden im Mittel die folgenden Zahlen gefunden. Um einen Vergleich zu haben,

1) Katz, Pflügers Archiv, Bd. 63, S. 1.

2) Voit, Zeitschr. f. Biologie, Bd. 35, S. 555. 
sind daneben die im Hundemuskel durch Katz bestimmten Werte angeführt:

$$
\text { Octopus }\left\{\begin{array}{ll}
\mathrm{Na}=1,5791 \mathrm{~g} & \mathrm{Na}=0,4000 \mathrm{~g} \\
\mathrm{~K}=2,0584, & \mathrm{~K}=1,4178, \\
\mathrm{Fe}=\text { unbestimmbare Spuren } & \mathrm{Fe}=0,0193, \\
\mathrm{Ca}=0,1226 \mathrm{~g} & \mathrm{Ca}=0,0291, \\
\mathrm{Mg}=0,2822, & \mathrm{Mg}=0,1005, \\
\mathrm{P}=0,4361, & \mathrm{P}=0,9478, \\
\mathrm{Cl}=2,7977, & \mathrm{Cl}=0,3415, \\
\mathrm{~S}=2,3913, & \mathrm{~S}=0,9643,
\end{array}\right\} \text { Hund }
$$

Die Zahlen fallen ganz aus dem Rahmen der bei den Wirbeltieren gefundenen Werte, trotzdem auch dort gar nicht so unbeträchtliche Schwankungen beobachtet worden sind. Eine eingehendere Gegenüberstellung dürfte zwecklos sein. Ein Überblick zeigt ohne weiteres die auffallenden Unterschiede.

Der Gehalt des Muskels an Alkalien und alkalischen Erden ist in seiner Gesamtheit bedeutend größer als bei den Wirbeltieren, und zwar zeigt sich ein Anwachsen sämtlicher Kationen. In Übereinstimmung damit steht der hohe Chlorgehalt. Die Konzentration der Muskelflüssigkeit an anorganischen Salzen ist demnach ungleich höher als bei den Wirbeltieren und nähert sich, wie aus anderen Erfahrungen über die osmotischen Verhältnisse des Blutes zu erwarten war, dem Milieu, in dem die Tiere leben. Es ist wohl zu beachten, der Grund für diese Erscheinung ist nicht darin $\mathrm{zu}$ suchen, daß die Tiere in einem salzreichen Milieu leben. Beim Schellfisch z. B. fallen die Werte der mineralischen Bestandteile des Muskels ganz in die Reihe der für die landbewohnenden Wirbeltiere gefundenen Werte.

Besonders hohe Zahlen zeigen die Natriumbestimmungen. Es läßt sich jedoch gleichzeitig damit auch eine Vermehrung der Kaliumsalze konstatieren, und zwar ist dieselbe so stark, daß auch im Octopusmuskel die letzteren den Natriumsalzen gegenüber überwiegen.

Der Phosphorgehalt ist im Vergleich zu den Wirbeltieren niedrig. Ganz auffallend hoch sind dagegen die Werte für Schwefel. Bei Carnivoren, zu denen der Octopus ja auch gehört, fand H. Schulz ${ }^{1}$ ) bei seinen Untersuchungen über den 
Schwefelgehalt tierischer Organe stets die höchsten Zahlen. Während im Muskel der höheren Wirbeltiere auf Trockensubstanz bezogen durchschnittlich $0,7-0,9 \%$ Schwefel gefunden wurden, stiegen die Schwefelwerte bei Fischen (Aal, Schellfisch) bis auf 1,1\% (Katz). Beim Octopus sehen wir den Schwefelgehalt bis auf $2,4 \%$ anwachsen. Der Taurinreichtum des Muskels fällt hier allerdings mit ins Gewicht, doch genügt dieser Faktor nicht, um diese Erscheinung zu erklären, wie man sich leicht durch eine Umrechnung überzeugen kann.

\section{Analytische Belege.}

Kalium und Natrium: Die Substanz wurde verkohlt, mit heißer verdünnter $\mathrm{HCl}$ ausgezogen, die Kohle mit etwas $\mathrm{H}_{2} \mathrm{SO}_{4}$ angerieben, getrocknet und verascht. Die Bestimmung der Alkalien erfolgte in der gewöhnlichen Weise.

I. Angew. Subst. $=4,5235 \mathrm{~g} \quad$ Ber. $\mathrm{KGl}=0,1878 \mathrm{~g}$

Gef. $\mathrm{NaCl}+\mathrm{KCl}=0,3684$, $, \mathrm{NaCl}=0,1806$, In Prozenten

- $\mathrm{K}_{2} \mathrm{PtCl}_{6}=0,6122$, Folgl. $\mathrm{K}=0,0986$, $\mathrm{Na}=1,5735$

$$
\text { , } \mathrm{Na}=0,0712, \mathrm{~K}=2,1803
$$

II. Angew. Subst. $=0,5720 \mathrm{~g}$ Ber. $\mathrm{KCl}=0,2421 \mathrm{~g}$

Gef. $\mathrm{NaCl}+\mathrm{KCl}=0,4945, \quad, \mathrm{NaCl}=0,2524$, In Prozenten

, $\mathrm{K}_{\mathrm{g}} \mathrm{PtCl}_{6}=0,7900$, Folgl. $\mathrm{K}=0,1273$, $\mathrm{K}=1,9365$

- $\mathrm{Na}=0,0995, \mathrm{Na}=1,5135$

III. Angew. Subst. $=0,0702 \mathrm{~g}$ Ber. $\mathrm{KCl}=0,1658 \mathrm{~g}$

Gef. $\mathrm{NaCl}+\mathrm{KCl}=0,4200$, , $\mathrm{NaCl}=0,2542$, In Prozenten

, $\mathrm{K}_{\mathrm{g}} \mathrm{PtCl}_{\mathrm{B}}=0,5400$, Folgl. $\mathrm{K}=0,0869, \mathrm{~K}=1,4329(?)$

, $\mathrm{Na}=0,1002$, $\mathrm{Na}=1,6503$

Mittel: $\mathrm{Na}=1,5791 \%$

, $\quad \mathrm{K}=2,0584 \%$ (aus I u. II).

Calcium und Magnesium: Die Substanz wurde unter Natriumkarbonatzusatz eingeäschert:

Angew. Substanz $=8,5048 \mathrm{~g}$

Gef. $\mathrm{CaO}=0,0146$,

Folgl. Ca $=0,0104$,

Gef. $\quad \mathrm{Mg}_{2} \mathrm{P}_{2} \mathrm{O}_{7}=0,1097 \mathrm{~g}$

In Prozenten $\mathrm{Ca}=0,1226 \%$ In Prozenten $\mathrm{Mg}=0,2822 \%$

1) H. Schulz, Pflügers Archiv, Bd. 54, S. 565. 
Phosphor: Der frische Muskel wurde mit Sodalösung eingetrocknet und verascht.

Angew. frischer Muskel $=80,0 \mathrm{~g}=18,154 \mathrm{~g}$ Trockensubstanz

Gef. $\quad \mathrm{Mg}_{2} \mathrm{P}_{2} \mathrm{O}_{7}=0,2870 \mathrm{~g}$

Folgl. $\mathrm{P}=0,0785$,

In Prozenten $\mathrm{P} \quad=0,0785 \%$

Chlor: Die Substanz wurde mit einer Lösung von $\mathrm{Ca}\left(\mathrm{NO}_{3}\right)_{2}$ eingetrocknet und im Silbertiegel verascht. Das Chlor wurde gewichtsanalytisch durch Fällung mit $\mathrm{AgNO}_{3}$ bestimmt.

Angew. Substanz $=4,8828 \mathrm{~g}$

Gef. $\mathrm{AgCl}=0,5520$,

Folgl. Cl $=0,1365$,

In Prozenten $\mathrm{Cl}=2,7977 \%$

Schwefel: Die von Katz empfohlene und von Klason herrührende Methode des Verbrennens der Substanz im Salpetersäurestrom konnte wegen mangelnder Platinapparate nicht benutzt werden. Es wurde deshalb so verfahren, daß die Substanz zunächst mit verdünnter, dann mit konzentrierter Salpetersäure auf dem Wasserbad zerstört wurde. Nach dem Verdampfen der Salpetersäure wurde der Rückstand unter Zusatz einer Lösung von Soda und etwas Salpeter eingetrocknet und vorsichtig verascht. Man bekommt auf diese Weise nur wenig Alkalisalze in die auszufällende Lösung. Das Baryumsulfat wurde trotzdem noch durch Lösen in konzentrierter Schwefelsäure und Ausfällen durch Verdünnen mit $\mathrm{W}^{\top}$ asser gereinigt.
I. Angew. Substanz $=1,9960 \mathrm{~g}$
Gef. $\mathrm{BaSO}_{4}=0,3280$, In Prozenten $\mathrm{S}=2,2612 \%$
Folgl. $\mathrm{S}=0,0451$ 。
II. Angew. Substanz $=4,6760 \mathrm{~g}$
Gef. $\mathrm{BaSO}_{4}=0,9616$, In Prozenten $\mathrm{S}=2,5214 \%$
Folgl. $\mathrm{S}=0,1302$,

\section{Zusammenfassung.}

Kurz zusammengefaßt ließen sich bisher folgende Tatsachen feststellen:

Der Octopusmuskel ist bei einem Wassergehalt von 77,3\% und Stickstoffgehalt von $13,13 \%$ ausgezeichnet durch seinen Taurinreichtum. 
Er enthält mindestens 0,5\% Taurin.

Kreatin oder Kreatinin fehlen im Muskel.

Die extrahierbaren Purinbasen scheinen fast ganz aus Hypoxanthin zu bestehen, und zwar in einer Menge von ca. 0,03\%.

Der Gesamtpurinbasenstickstoff beträgt $0,0456 \%$.

Es fehlen Harnstoff, Hexonbasen und Amidosäuren, speziell Glykokoll.

Eine unbekannte Base konnte in Form ihres Platinsalzes isoliert, aber bisher nicht näher charakterisiert werden.

Der Ammoniakgehalt des Muskels beträgt in Milligrammen $7,48 \%$.

Fleischmilchsäure fehlt dem Muskel offenbar ganz, dagegen wurden sehr geringe Mengen $(0,01 \%)$ Gärungsmilchsäure gefunden.

Einen Reservestoff in Form von Glykogen kann der Muskel nicht enthalten.

Der Octopusmuskel ist ungleich reicher an anorganischen Salzen als der Wirbeltiermuskel.

Die Kaliumsalze überwiegen trotz des Reichtums an Natriumsalzen.

Auffallend ist der hohe Schwefelgehalt des Muskels, der etwa dreimal so groß ist als bei den Wirbeltieren. 